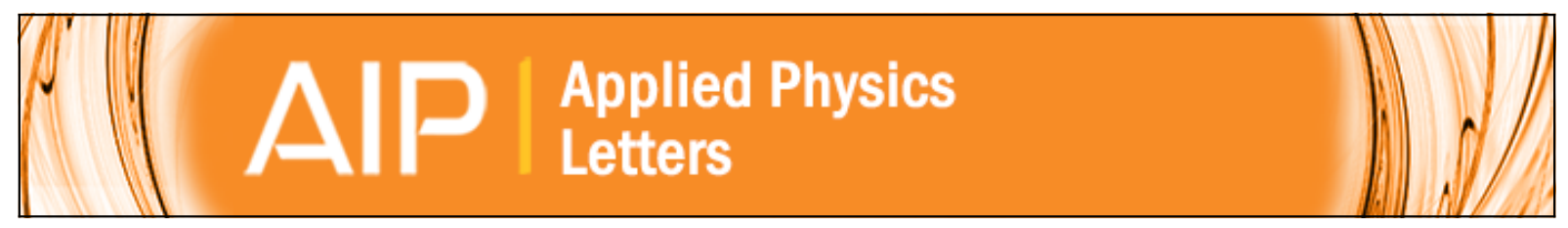

\title{
Spatially resolved photoluminescence mapping of single CdS nanosheets
}

H. Rho, K.-Y. Lee, T. B. Hoang, L. V. Titova, A. Mishra, L. M. Smith, H. E. Jackson, J. M. Yarrison-Rice, Y.-J.

Choi, K. J. Choi, and J.-G. Park

Citation: Applied Physics Letters 92, 013111 (2008); doi: 10.1063/1.2828707

View online: http://dx.doi.org/10.1063/1.2828707

View Table of Contents: http://scitation.aip.org/content/aip/journal/apl/92/1?ver=pdfcov

Published by the AIP Publishing

\section{Articles you may be interested in}

Raman stress mapping of CdS nanosheets

Appl. Phys. Lett. 95, 083105 (2009); 10.1063/1.3211121

Polarized photoluminescence and time-resolved photoluminescence from single CdS nanosheets

Appl. Phys. Lett. 92, 143112 (2008); 10.1063/1.2907507

Low-temperature photoluminescence imaging and time-resolved spectroscopy of single CdS nanowires

Appl. Phys. Lett. 89, 053119 (2006); 10.1063/1.2266414

Photoluminescence properties of single Mn-doped CdS nanocrystals studied by scanning near-field optical microscopy

Appl. Phys. Lett. 87, 133104 (2005); 10.1063/1.2058228

Photoluminescence spectrum of highly excited single CdS nanocrystals studied by a scanning near-field optical microscope

Appl. Phys. Lett. 81, 141 (2002); 10.1063/1.1490141

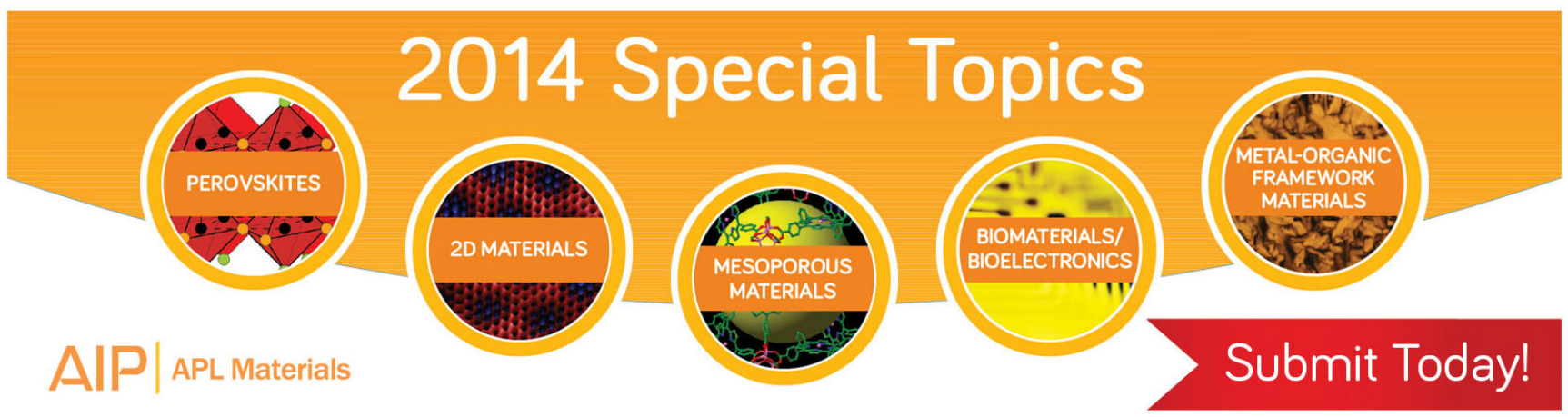




\title{
Spatially resolved photoluminescence mapping of single CdS nanosheets
}

\author{
H. Rho ${ }^{\mathrm{a}), \mathrm{b})}$ and K.-Y. Lee \\ Department of Physics, Chonbuk National University, Jeonju 561-756, Korea \\ T. B. Hoang, L. V. Titova, A. Mishra, L. M. Smith, and H. E. Jackson ${ }^{\text {a),c) }}$ \\ Department of Physics, University of Cincinnati, Cincinnati, Ohio 45221, USA \\ J. M. Yarrison-Rice \\ Physics Department, Miami University, Oxford, Ohio 45056, USA \\ Y.-J. Choi, K. J. Choi, and J.-G. Park \\ Nano Materials Research Center, Korea Institute of Science and Technology, Seoul 130-650, Korea
}

(Received 16 October 2007; accepted 5 December 2007; published online 4 January 2008)

\begin{abstract}
We have utilized spatially resolved low temperature photoluminescence to probe the electronic states and structural symmetries of individual single crystalline CdS nanosheets. Spatially resolved photoluminescence imaging of a single $\mathrm{CdS}$ nanosheet reveals a distinctive spectral variation across the nanosheet. We observe $A$-like exciton states which emit most strongly near the outer edges of the $5 \mu \mathrm{m}$ wide nanosheet, while $B$-like exciton states emit most strongly along the center region of the $30 \mu \mathrm{m}$ long axis of the nanosheet. (C) 2008 American Institute of Physics.
\end{abstract}

[DOI: $10.1063 / 1.2828707$ ]

Semiconductor nanostructures ranging from quantum dots to nanowires to nanosheets have recently attracted considerable attention because of the interesting physics in reduced dimensions, the spatially confined optical and electrical properties, and a plethora of potential applications. In particular, semiconductor nanosheets with good crystallinity are poised to support applications in nanoscale electronic and optoelectronic devices. $^{1-9}$ The last few years have seen a variety of different methods used to produce single crystalline nanobelts or nanosheets of materials including $\mathrm{ZnO},{ }^{10}$ $\mathrm{SnO}_{2},{ }^{11} \mathrm{Ga}_{2} \mathrm{O}_{3},{ }^{12} \mathrm{MgO},{ }^{13} \mathrm{ZnS},{ }^{14}$ and $\mathrm{CdS} .{ }^{8,15} \mathrm{CdS}$ nanosheets reported here present significant opportunities for optoelectronic applications with optical switches, high quality field effect transistors, and light emitting diodes recently being demonstrated. ${ }^{1,5,6,9}$ Further development of these applications should clearly benefit from a deeper understanding of the nanosheet optical and electronic properties.

In this letter, we use low temperature microphotoluminescence in combination with spatially resolved photoluminescence (PL) spectroscopy to investigate the optical properties of single $\mathrm{CdS}$ nanosheets. High resolution transmission electron microscope (HRTEM) images show single crystal, high quality nanosheet growth with hexagonal wurtzite structure. The $c$ axis is seen to be approximately perpendicular to the nanosheet long axis. ${ }^{16}$ Using low-temperature polarized PL imaging, we observe high quantum efficiency emission corresponding to $A$ - and $B$-hole band excitons and, through polarization analysis, we can determine the orientation of the $c$ axis for a particular single $\mathrm{CdS}$ nanosheet. ${ }^{16}$ Spatially resolved PL imaging of single nanosheets reveals striking PL intensity and line shape variation along the length and width of a nanosheet. This implies some variation in uniformity, strain, or defects across the nanosheet. All nanosheet dimensions $(\sim 50 \mathrm{~nm}$ thickness, $\sim 5 \mu \mathrm{m}$ width, and $\sim 30 \mu \mathrm{m}$ length) are much larger than the $\mathrm{CdS}$ Bohr exciton radius

\footnotetext{
a) Authors to whom correspondence should be addressed.

${ }^{b)}$ Electronic mail: rho@chonbuk.ac.kr.

${ }^{c)}$ Electronic mail: howard.jackson@uc.edu.
}

(6 nm) and, thus, no quantum confinement effects are expected. CdS nanosheets were grown by pulsed laser deposition using the vapor-phase transport method. ${ }^{17}$

The size and shape of the nanosheets are quite uniform. The upper inset in Fig. 1 shows a high-magnification scanning electron microscopy (SEM) image of a single nanosheet. The surface of the nanosheet is curved and smooth with a uniform thickness over the larger surface area. To measure single structures, the nanosheets were dispersed from the growth substrate into an ethanol solution and then deposited on a silicon substrate; the as-grown nanosheets (see lower inset of Fig. 1) are held flat to the substrate by van der Waals' forces.

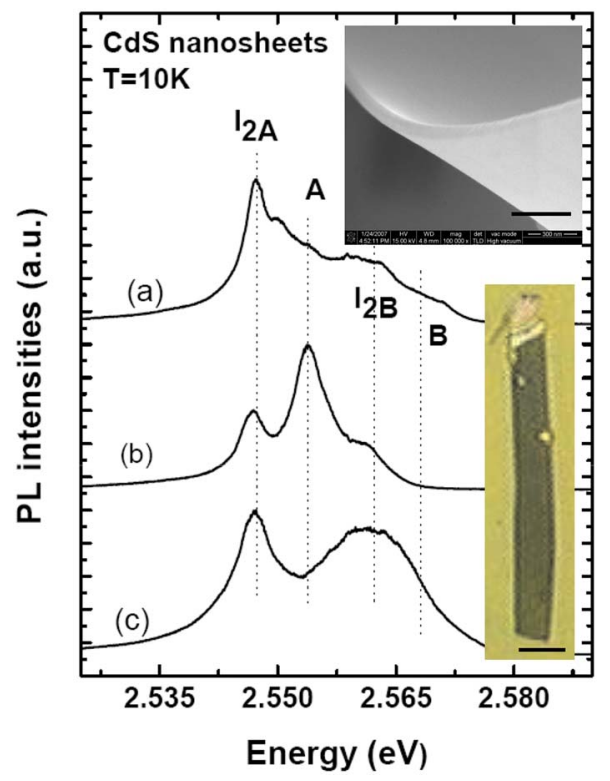

FIG. 1. (Color online) Normalized photoluminescence spectra from three different single nanosheets. Upper inset is a high-magnification SEM image of a single nanosheet (dark line $=300 \mathrm{~nm}$ ) and lower inset is an optical micrograph of the $\sim 5 \times 30 \mu \mathrm{m}^{2}$ nanosheet. 
The crystallinity and structure of individual CdS nanosheets were investigated by HRTEM which showed a highly crystalline wurtzite structure with the $c$ axis nearly perpendicular to the long axis of the CdS nanosheet. Other $\mathrm{CdS}$ nanosheets, however, were found to have the $c$ axis at other orientations relative to the long axis of the nanosheet. ${ }^{16}$ Low temperature PL from a single nanosheet was collected using a $50 \times / 0.5$ numerical aperture long working length microscope objective, which was also used to focus the $458 \mathrm{~nm}$ line of a cw Argon ion laser. The PL was dispersed by a triple spectrometer working in subtractive mode, and detected by a $2000 \times 800$ pixel liquid nitrogen-cooled charge-coupled device (CCD) detector. Spectra were measured with the excitation laser circularly polarized while the emission passed through a linear polarization analyzer aligned parallel to the long axis of each nanosheet. All measurements were conducted at $15 \mathrm{~K}$.

In Fig. 1, we show typical low temperature cw PL spectra of three different single CdS nanosheets. The PL intensity from each nanosheet is strong, indicating emission from the $\mathrm{CdS}$ nanosheet occurs with high quantum efficiency. This emission is entirely near the CdS band edge and, unlike previous CdS nanosheet low temperature PL measurements, no evidence for donor-acceptor or surface related emission was seen at lower energies. ${ }^{4,18}$ We find that each of the nanosheets displays a narrow peak [full width at half maxi- mum (FHWM) $\sim 4 \mathrm{meV}]$ at $2.547 \mathrm{eV}$ and a broader response (FHWM $\sim 11 \mathrm{meV}$ ) at higher energies near $2.563 \mathrm{eV}$. The broad response varies in energy $(2.553-2.571 \mathrm{eV})$ from sheet to sheet and can also display internal spectral structure indicating contributions from more than one exciton state. Recall that in the wurtzite structure the $p$-like hole bands are split so that there may be three excitons labeled $A, B$, and $C$. The lower energy peak we observe is situated near the spectral line observed in bulk $\mathrm{CdS}$ associated with the $I_{2 A}$ line at $2.547 \mathrm{eV}$, which results from recombination of an $A$ exciton bound to a neutral donor. The $A$ free exciton should be some $6 \mathrm{meV}$ higher in energy at $2.553 \mathrm{eV} .^{19,20}$ The higher energy broad peak is in the region of the $I_{2 B}$ line at $2.563 \mathrm{eV}$, resulting from recombination of a $B$ exciton bound to a neutral donor. The $B$ free exciton line is expected at $2.569 \mathrm{eV}$.

To gain more insight into the nature of these two lines, which exist in the $A$ - and $B$-excitonic regions for $\mathrm{CdS}$, we use slit-confocal microscopy to directly image an entire $\mathrm{CdS}$ nanosheet with $1.5 \mu \mathrm{m}$ spatial resolution. This technique is described in more detail in Hewaparakrama et al. ${ }^{21}$ The nanosheet, with its entire area excited by a defocused laser, is scanned underneath the fixed microscope objective, and a series of two-dimensional CCD spectral images are collected. Ultimately, a large three-dimensional data set is acquired, which has full 500 point spectra at every spatial point
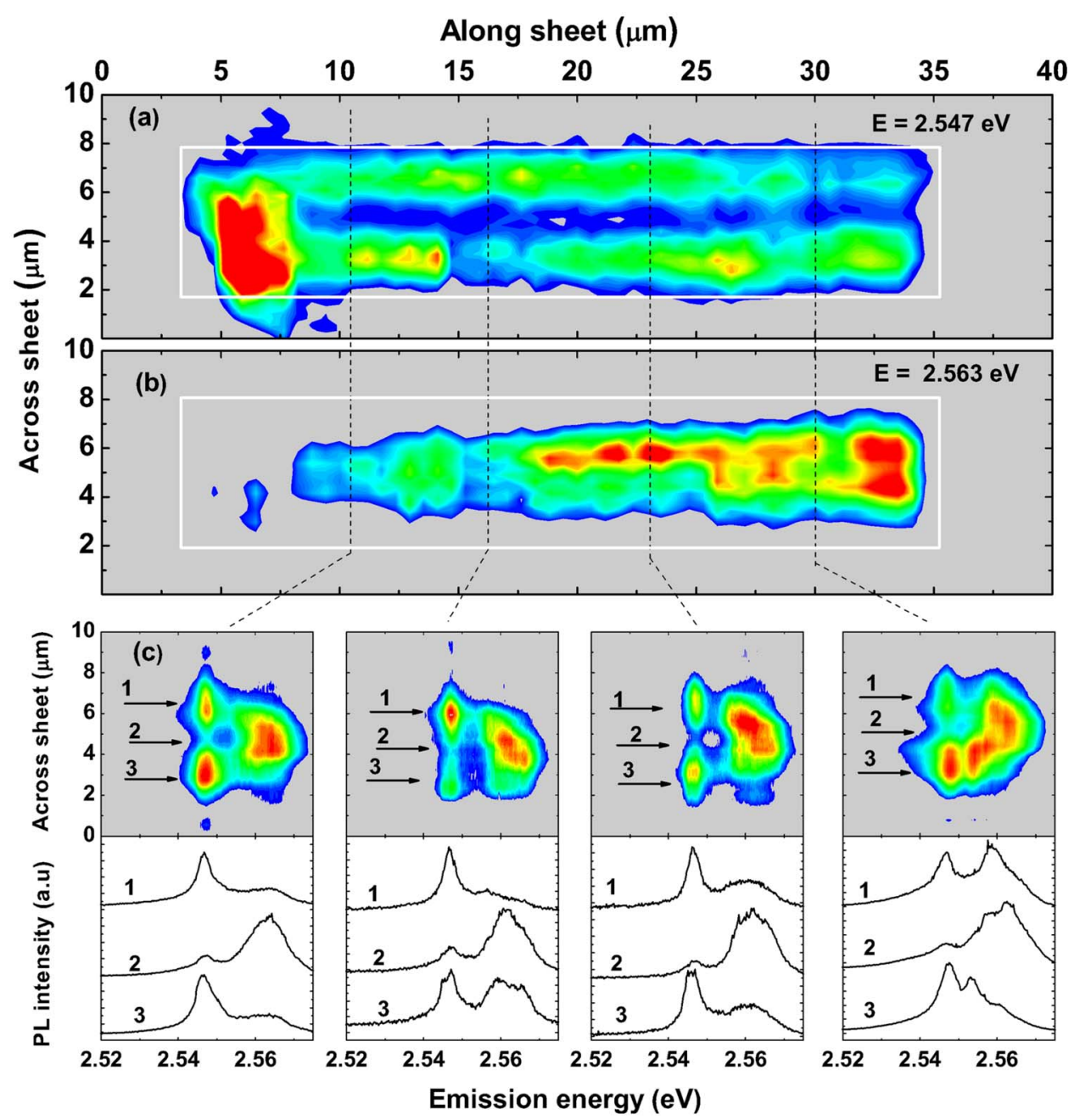
along and across the nanosheet. In this way, it becomes possible to correlate spectral and spatial features of the nanosheet.

We present in detail such spatially resolved PL measurements of nanosheet 3 in Fig. 2. This nanosheet is $\sim 5 \mu \mathrm{m}$ wide $\times 30 \mu \mathrm{m}$ long. Figures 2(a) and 2(b) show PL images of this nanosheet at two photon energies (2.547 and $2.563 \mathrm{eV}$ ) associated with the two well-resolved spectral peaks seen for the nanosheet in Fig. 1(c) for nanosheet 3. These spatial maps aredisplayed as false-color images on a logarithmic scale with red used for the most intense emission from the nanosheet. In these images, the vertical and horizontal axes denote the spatial positions across the width and along the length of the nanosheet, respectively. Quite unexpectedly, we find that, as displayed in Fig. 2(a), the peak at lower energy emits most strongly along the edges of the nanosheet, while, as seen in Fig. 2(b), the higher energy peak emits most strongly at the center of the nanosheet. From both the spatial maps and the selected area PL spectra, we observe that the emissions which we assign to the $I_{2 A}$ and $I_{2 B}$ donor bound excitons emit in different spatial regions of the nanosheet.

A plausible explanation of this distinctive spatial separation may be the spatial distribution of stress in this system. SEM images show that stress induces the curl of the nanosheets and that the flattening onto a substrate for optical measurement may induce stress. Recent Raman measurements ${ }^{22}$ also indicate the presence of significant stress in the nanosheets. A spatial gradient of stress from the center to the edge of the nanosheet would affect the $A$ and $B$ excitons differentially, ${ }^{23}$ resulting in potential minima either along the edge or in the center for the $A$ - and $B$-valence band excitons, respectively. More detailed measurements with the higher spatial resolution offered by use of a solid immersion lense are clearly required to interpret these results. ${ }^{21,24}$

To display more detailed spectral information along the nanosheet, we extract the original CCD spectral maps from the full three-dimensional data set. These spectral maps, shown in the bottom row of images of Fig. 2, display the PL spectra as a function of position across the width of the nanosheet. Four such spectral maps are shown at positions along the length of the nanosheet, as shown by the dashed lines. From these four spectral maps in the bottom row of Fig. 2(c) a number of general observations can be made. We see that in every cross section the spectral peak at $2.547 \mathrm{eV}$ appears most intensely at the edges of the nanosheet. Moreover, the broader peak observed at the higher energy of $\sim 2.563 \mathrm{eV}$ predominantly emits along the center of the nanosheet, but the spectral position of this peak appears to vary both in energy and position from slice to slice along the nanosheet. Below each spectral map are shown PL emission spectra extracted from three different vertical locations as indicated by the horizontal arrows in each spectral map. From such spatially resolved spectra, we can see that the PL spectrum not only changes when crossing the width of the nanosheet, but also depends on position along the length of the nanosheet. Other nanosheets showed similar PL imaging results.

In conclusion, we have shown that catalyst-assisted vapor phase growth of $\mathrm{CdS}$ nanosheets with large width-tothickness aspect ratios results in high quality hexagonal wurtzite single crystals which display intense band edge emission at low temperatures with no noticeable defect emission at lower energies. Both $A$ - and $B$-related exciton emissions are observed, with spatially resolved PL spectra of single nanosheets showing striking spatial variability of the intensity of these lines across the nanosheet indicating the influence of strains. Finally, we have shown how spatially resolved low temperature PL spectroscopy can rapidly characterize the electronic and crystalline structure of nanosheets in a nondestructive way.

H.R., L.V.T., and T.B.H. are equally responsible for this work. This work was supported by the National Science Foundation by Grant Nos. EEC/NUE 0532495 and ECCS 0701703, USA, by the Korea Research Foundation Grant (KRF-2006-005-J00302), by Brain Korea 21 by Chonbuk National University, 2007, and the nanosheets growth by the KIST-CNRS LIA program (2U03450) and the KIST project (2E19770).

${ }^{1}$ X. F. Duan, C. M. Niu, V. Sahi, J. Chen, J. W. Parce, S. Empedocles, and J. L. Goldman, Nature (London) 425, 274 (2003).

${ }^{2}$ Z. L. Wang, Annu. Rev. Phys. Chem. 55, 159 (2004).

${ }^{3}$ L. Weifeng, J. Chong, J. Chuangui, Y. Lianzeng, C. Weili, and L. Xiaoguang, J. Cryst. Growth 269, 304 (2004).

${ }^{4}$ W. Chunrui, K. M. Ip, S. K. Hark, and L. Quan, J. Appl. Phys. 97, 054303 (2005).

${ }^{5}$ T. Gao, Q. H. Li, and T. H. Wang, Appl. Phys. Lett. 86, 173105 (2005).

${ }^{6}$ R. M. Ma, L. Dai, H. B. Huo, W. Q. Yang, G. G. Qin, P. H. Tan, C. H. Huang, and J. Zheng, Appl. Phys. Lett. 89, 203120 (2006).

${ }^{7}$ D. H. L. Ng, W. Yu, and C. Y. To, Mater. Lett. 60, 1151 (2006).

${ }^{8}$ Z. Q. Wang, J. F. Gong, J. H. Duan, H. B. Huang, S. G. Yang, X. N. Zhao, R. Zhang, and Y. W. Du, Appl. Phys. Lett. 89, 033102 (2006).

${ }^{9}$ R. M. Ma, L. Dai, and G. G. Qin, Nano Lett. 7, 868 (2007).

${ }^{10}$ P. Z. Wei, D. Z. Rong, and W. Z. Lin, Science 291, 1947 (2001).

${ }^{11}$ S. H. Sun, G. W. Meng, G. X. Zhang, T. Gao, B. Y. Geng, L. D. Zhang, and J. Zuo, Chem. Phys. Lett. 376, 103 (2003).

${ }^{12}$ Z. R. Dai, Z. W. Pan, and Z. L. Wang, J. Phys. Chem. B 106, 902 (2002).

${ }^{13}$ R. Ma and Y. Bando, Chem. Phys. Lett. 370, 770 (2003).

${ }^{14}$ Y.-C. Zhu, Y. Bando, and D.-F. Xoe, Appl. Phys. Lett. 82, 1769 (2003).

${ }^{15}$ L. Chen, L. Zengtao, and Y. Yong, Nanotechnology 17, 1851 (2006).

${ }^{16}$ T. B. Hoang, H. Rho, L. V. Titova, A. Mishra, K.-Y. Lee, J. M. YarrisonRice, H. E. Jackson, L. M. Smith, Y.-J. Choi, K. J. Choi, and J.-G. Park "Polarized photoluminescence and time-resolved photoluminescence from single CdS nanosheets," (unpublished).

${ }^{17}$ M. H. Huang, Y. Y. Wu, H. Feick, N. Tran, E. Weber, and P. D. Yang, Adv. Mater. (Weinheim, Ger.) 13, 113 (2001).

${ }^{18}$ K. M. Ip, C. R. Wang, Q. Li, and S. K. Hark, Appl. Phys. Lett. 84, 795 (2004).

${ }^{19}$ D. G. Thomas and J. J. Hopfield, Phys. Rev. 128, 2135 (1962).

${ }^{20}$ J. J. Hopfield and D. G. Thomas, Phys. Rev. 122, 35 (1961).

${ }^{21}$ K. P. Hewaparakrama, A. Wilson, S. Mackowski, H. E. Jackson, L. M. Smith, G. Karczewski, and J. Kossut, Appl. Phys. Lett. 85, 5463 (2004).

${ }^{22}$ K.-Y. Lee, J.-R. Lim, H. Rho, Y.-J. Choi, K. J. Choi, and J.-G. Park, Appl. Phys. Lett. 91, 201901 (2007).

${ }^{23}$ D. W. Langer, R. N. Euwema, K. Era, and T. Koda, Phys. Rev. B 2, 4005 (1970).

${ }^{24}$ S. Mackowski, T. Gurung, H. E. Jackson, L. M. Smith, G. Karczewski, and J. Kossut, Appl. Phys. Lett. 87, 072502 (2005). 